\title{
Motivated Gaze
}

\section{The View From the Gazer}

\author{
Derek M. Isaacowitz
}

Brandeis University

\begin{abstract}
How does gaze relate to psychological properties of the gazer? Studies using eye tracking reveal robust group differences in gaze toward emotional information: Optimists gaze less at negative, unpleasant images than do pessimists, and older individuals look away from negative faces and toward happy faces. These group differences appear to reflect an underlying motivation to achieve and maintain good moods by directing attention to moodfacilitating stimuli. Maintaining a positive mood is only one goal-related context that influences visual attention; recent work has also suggested that other goal states can impact gaze. Gaze therefore is a tool of motivation, directing gazers toward stimuli that are consistent with their goals and away from information that will not facilitate goal achievement.
\end{abstract}

KEYWORDS_attention; motivation; optimism; aging

References to how gaze can be used strategically abound in everyday conversation: For example, people commonly advise distressed friends to "look on the bright side" of a bad situation. Similarly, the survivor of a traumatic experience may describe having new "perspective" on life since the event. While perhaps meant figuratively, such comments may have some literal truth. Is it possible that gaze can actually be used strategically by gazers?

In this article, I propose several ways of linking gaze to psychological properties of the gazer, as a step toward developing a general model of how motivation regulates visual attention. First, gaze is linked both to individual differences in personalityrelated constructs and to individual development. Second, gaze is a tool for optimizing the affect of the gazer. Third, gaze serves a general motivational role, guiding people toward information that will help them achieve goals and away from stimuli that will not.

Address correspondence to Derek M. Isaacowitz, Department of Psychology, Brandeis University, MS 062, Waltham, MA 02454-9110; e-mail: dmi@brandeis.edu.
I describe results of studies using eye tracking to measure gaze directly, in nearly real time, to support these general properties of gaze. I also use research using observation of gaze, when it is available, to support eye-tracking findings. While it is possible to separate gaze from visual attention, the two are generally considered identical (Parkhurst, Law, \& Niebur, 2002); thus, here I use gaze to refer to where an individual fixates, but it could also be called the target of visual attention.

\section{GAZE IS TIED TO PERSONALITY AND DEVELOPMENT}

In my lab, we have used eye tracking, a technique previously used mostly in reading research, to investigate group differences in visual attention to emotional stimuli. Participants are seated in front of a computer screen; a remote eye-tracking system records their left-eye gaze position using infrared illumination 60 times a second. Fixations are defined as gaze within a small area for at least 100 milliseconds, allowing us to distinguish between saccades (fast eye movements between fixations) and gaze meaningfully fixated on stimuli. Using fixation to emotional stimuli as the dependent variable, several robust group differences in gaze have been documented.

One eye-tracking study conducted in my lab tested for differences in gaze patterns among young adults with different levels of dispositional optimism as indicated by a self-report optimism measure (Isaacowitz, 2005, Study 1), evaluating whether some individuals can indeed look on the bright side of things. Participants' eyes were tracked as they viewed images of skin cancer, line drawings with the same shape as the cancer images, and neutral faces. Skin cancer was selected because it had been used in past work on optimism-health relations and was unpleasant as well as graphic. Relatively optimistic young adults fixated less on the skin-cancer images than their less optimistic peers did, even in a conservative test in which fixation to schematic drawings (matched for lines and shapes to the cancer images but without the actual unpleasant cancer component) was controlled. This effect of optimism was not eliminated by controlling for affect (i.e., participants' mood at the time of the test) or neuroticism, nor did a perceived-relevance index 
of family history and worry about skin cancer interact with optimism.

This evidence of optimists wearing "rose-colored glasses" was provocative, so I conducted a follow-up study to replicate and extend the findings (Isaacowitz, 2005, Study 2). The setup was similar, with two additions. First, participants completed selfreport anxiety measures to rule out the possibility that observed effects were simply due to anxiety (as anxious individuals appear especially vigilant toward potentially threatening stimuli). Second, self-relevance was further examined through an experimental manipulation: Some participants were told that they were going to see images related to real-world health concerns and problem detection (relevance condition), whereas others were asked to "look naturally" as if at home watching television (control). The effect of optimism on visual attention to the skin cancer was replicated, and neither the relevance manipulation nor anxiety predicted gaze. Again, more optimistic young adults looked less at the skin cancer than their more pessimistic peers did. In both studies, no effect of expecting in advance to see skincancer images was found: Gaze patterns were similar for cancer stimuli both early and late in the presentation.

We moved even closer to real-world health-related attention in another study, when we measured the gaze of college students as they viewed text and pictorial skin-cancer information (Luo \& Isaacowitz, 2006). We followed Aspinwall and Brunhart's (1996) approach of defining relevance by how much risk each participant possessed for the particular negative outcome; however, unlike the self-reported risk information used by Aspinwall, we used an objective risk-assessment tool that indexed actual risk factors for skin cancer to determine risk relevance for each participant. Results for dispositional optimism replicated the previous findings: Individuals high in dispositional optimism appeared to look less at cancer-related stimuli, even in some cases when the stimuli were objectively relevant to them. ${ }^{1}$ These three studies therefore point consistently to individuals higher in dispositional optimism looking less at unpleasant stimuli, regardless of the self-relevance (defined in various ways) of those stimuli.

My lab has also been studying gaze and aging, pursuing theoretical work suggesting that emotional material may be particularly salient to older adults (Carstensen, Isaacowitz, \& Charles, 1999). In the first eye-tracking study to investigate agerelated gaze preferences for different emotional stimuli (Isaacowitz, Wadlinger, Goren, \& Wilson, in press), synthetic faces displaying emotional and nonemotional expressions were used as target stimuli. These faces minimized psychophysical

\footnotetext{
${ }^{1}$ Aspinwall and Brunhart (1996) found that health-related optimism predicted longer latency to click off a Web page featuring self-relevant negative information. In Luo \& Isaacowitz (2006), participants who were high in dispositional optimism but low in health-related optimism looked less at health-related information as self-relevance increased. This dissociation between health-specific optimism and dispositional optimism is beyond the scope of this article but warrants future research.
}

differences that might grab attention while simultaneously using facial geometry to create expressions of happiness, sadness, anger, and fear. Pretest ratings ensured that the faces did indeed represent the intended emotional expression and were equivalent in intensity across emotional subtypes. Participants had their eyes tracked as they "looked naturally" at images featuring a face making an emotional expression paired with the same face in a nonemotional expression. Gaze-preference scores were used to explore whether a particular age group showed a pattern of looking toward or away from the emotional faces in those pairs. Older participants (age 57-84) showed a gaze preference toward happy and away from angry faces. Younger adults' (age 18-21) only preference was toward afraid faces. An example of these gaze preferences is shown in Figure 1. This is evidence for specific attentional preferences in both young and older adults; importantly, these preferences were found in a sample in which the age groups were well matched in overall cognitive functioning, and the effects were not eliminated when statistically controlling for perceptual individual-difference variables, such as visual acuity. Older adults show gaze preferences toward positive and away from negative emotional stimuli in their environment.

\section{GAZE IS A TOOL FOR OPTIMIZING POSITIVE AFFECT}

Findings of optimists gazing less at negative images and of older adults showing gaze preferences toward positive and away from negative images suggest that gaze patterns may reflect an underlying motivation to regulate emotions and to feel good. There is theoretical rationale for a motivational basis for these "feel good" patterns of attention, at least in terms of age-related patterns. According to socioemotional selectivity theory (Carstensen et al., 1999), contexts in which time is perceived as limited (such as old age) are associated with motivation to pursue emotionally meaningful goals and to regulate emotional experience in a positive direction. The evidence presented earlier, that older individuals show gaze preferences favoring positive material, is consistent with a motivation to process information to optimize positive affect. However, such age differences may reflect more general effects of cognitive aging rather than specifically motivated processes.

Several approaches have been used to ensure that age differences in gaze reflect motivation to feel good rather than artifacts of cognitive aging. The first involves extensive perceptual and cognitive testing, then using matching or statistical controls to show that gaze patterns are not due to these more general changes in perception and cognition that occur with age (e.g., Isaacowitz et al., in press). Second, a study was conducted in my lab comparing gaze toward emotional faces in two groups that are generally comparable in cognitive functioning but vary in time perspective: first-year college students and seniors. College seniors, for whom impending graduation served to constrain time perspective, gazed less at negative 

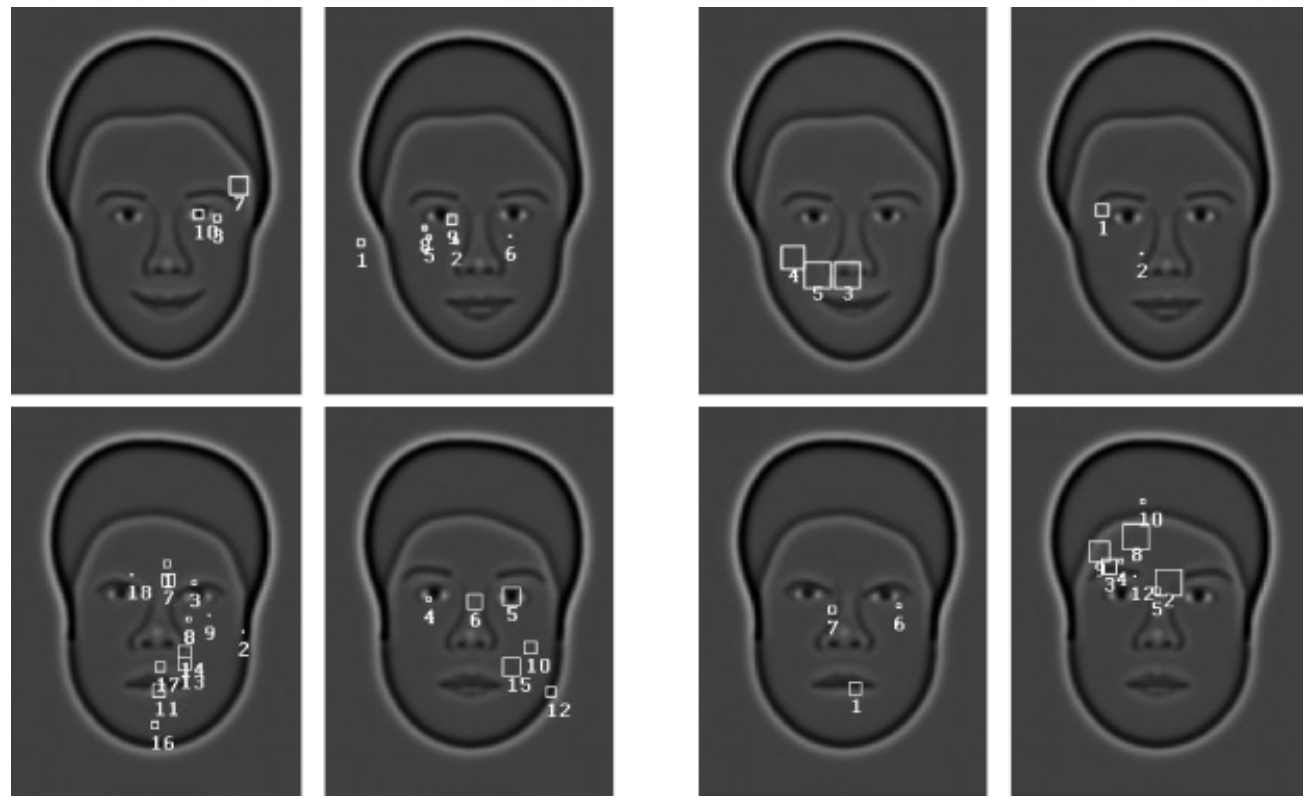

Fig. 1. Gaze-fixation patterns of a younger adult (left group of panels) and an older adult (right group of panels) to the same face images for a happy-neutral (top) and angry-neutral (bottom) pair. Numbers signify order of fixation ( 1 = first fixation), and box size is an index of fixation length, with larger boxes indicating a longer fixation at that location. Percent fixation within the Area of Interest (AOI) for the happy-neutral pair (top panels) was $43.21 \%$ to the happy face and $47.09 \%$ to the neutral face for the younger individual and $78.62 \%$ to the happy face and $15.18 \%$ to the neutral face for the older individual. Percent fixation within the AOI for the angry-neutral pair (bottom panels) was $54.37 \%$ to the angry face and $45.63 \%$ to the neutral face for the younger individual and $14.49 \%$ to the angry face and $85.51 \%$ to the neutral face for the older individual. From Isaacowitz, Wadlinger, Goren, and Wilson (in press).

stimuli than did first-year students. Consistent with gaze preferences relating to affect, the college seniors were also happier (Pruzan \& Isaacowitz, 2006). Together, these findings suggest that motivational rather than cognitive factors influence observed gaze patterns.

An even more powerful demonstration that motivation to feel good can lead to observed gaze patterns comes from a recent study of young adults randomly assigned to instruction conditions mimicking motivational states before viewing emotionally valenced images (Xing \& Isaacowitz, 2006). Some participants were instructed to regulate their emotions as they viewed (the instruction was to "manage how you feel as you view"), whereas others were told to gather as much information from the pictures as possible. Participants instructed to regulate emotions gazed less at negative than at positive or neutral stimuli, whereas no such differences were found among those told to gather information.

It is worth noting that a motivation to achieve and maintain positive mood by selective information processing is not unique to socioemotional selectivity theory; the hedonic contingency model (Wegener \& Petty, 1994) also proposes motivated information processing in the service of positive mood maintenance. Thus, different motivational theories share the same prediction, supported by eye-tracking evidence, that gaze can work to keep the gazer in a positive mood.

\section{GAZE IS A GENERAL TOOL OF MOTIVATION AND GOAL-DIRECTED BEHAVIOR}

The above evidence suggests that gaze is a tool of optimizing affect; however, feeling good is only one goal that people may be motivated to accomplish. The final proposition of this paper is that gaze serves as a more general tool of motivation. For example, the life-span theory of control (Heckhausen \& Schulz, 1995) suggests that the underlying motivation for humans is to maintain control over their environment. In the context of development, that means focusing resources on goal achievement when a goal is still possible and disengaging from goals that are no longer achievable (due to biological changes, for example). Pursuing unattainable goals might lead to unhappiness, but the theory argues that continuing engagement with goals that cannot be attained does more than just make people unhappy; it also impairs the ability to exert and maintain control in other domains. Thus, emotional ramifications are secondary to motivational and behavioral ones. My lab set out to investigate whether gaze could be linked to this theoretical perspective on motivation.

Jessica Light and I (Light \& Isaacowitz, in press) adopted a paradigm from control research that examines processing of goal-relevant and goal-irrelevant stimuli in individuals who are before a developmental deadline and in those who have passed 
it. This was done by investigating attention among childless women who were either before or after the developmental deadline for childbearing. While technological advances have allowed some females to extend their childbearing years, there continues to be a shared perception of the age (around 40) at which having children becomes significantly less likely. We measured gaze patterns as participants viewed images of babies and pictures of puppies and kittens matched for cuteness. Postdeadline participants averted their gaze from babies faster than did pre-deadline women, while the groups did not differ in gaze toward non-baby stimuli. Gaze appeared to be more closely tied to goal processes than to affective outcomes, supporting the idea that gaze can reflect more general motivational processes than emotion regulation. Moreover, the life-span theory of control would argue that any affective results of motivated information processing are adaptive only if they facilitate achievement of behavioral control; gaze reflecting control motivation should therefore be oriented toward stimuli that optimize control.

Earlier work using observational methods supports the idea that gaze works in the general service of goal achievement, sometimes even at the expense of feeling good. Kleinke (1986) reviews findings in which individuals gaze more at information that will not make them feel good-such as looking more at a person who has given you negative feedback in order to determine why. Together with our work on gaze toward baby pictures, these findings suggest that gaze does not orient only to happymaking stimuli in the environment, and even when gaze is directed toward positive material, it may be working in support of longer-term general goals rather than short-term emotional ones.

Given that motivated cognition has been documented in other stages of information processing, such as reasoning (Kunda, 1990), why is another tool needed in the toolbox of motivated cognition? Successful goal pursuit likely requires multiple levels of cognitive effort, due to the number of intruding non-goal-relevant stimuli in the environment. Someone with the goal of feeling good will be assaulted with negative images on the evening news, and someone trying to get a task done may get interrupted by competing demands, such as receiving a distracting e-mail. Older adults may be at even greater risk of goal-irrelevant stimuli impinging on their thinking. Thus, multiple levels of motivated cognition are needed. Motivational influences on gaze are particularly efficient at keeping goal-irrelevant stimuli out of later processing: Gaze is highly selective, happens early in processing, and appears responsive to multiple motivational influencesfrom maintaining control to feeling good. Gaze is thus perhaps the most economical tool in motivation's toolbox.

\section{FUTURE DIRECTIONS}

The findings I discussed suggest important basic issues that might be investigated using gaze, such as whether the motivational effects on gaze are automatic or consciously controlled. For example, if individuals can force themselves to look on the bright side of things, can that volitional process be transformed into an automatic one with repeated intentional practice?

Time is obviously critical when it comes to interpreting gaze data. While fixations may vary from 100 to 500 milliseconds in length (or longer), it takes 150 to 175 milliseconds to execute an eye movement (Reichle, Pollatsek, Fisher, \& Rayner, 1998). Millisecond-by-millisecond analyses of our gaze data are currently underway. It is nonetheless notable that the earliest effect of motivation we have found on gaze has been on duration of first fixation (the first location on the image on which the participant fixates); we have not documented any effects thus far on the very earliest aspects of gaze (such as where the first fixation falls). This pattern suggests that some visual scanning is occurring before motivated effects on gaze pattern emerge, which is reasonable given that some basic detection must take place to provide input for motivation to act on. This interpretation is supported by recent findings that older adults detect angry stimuli similarly to younger adults (Mather \& Knight, 2006) but avert their gaze away from such stimuli over longer time intervals (Isaacowitz et al., in press). The search for the mechanisms underlying motivated-gaze effects will require attention to time; motivational effects are likely rapid, but not immediate.

Another future direction involves determining the behavioral effects of motivated gaze. We have started to document connections between motivation, gaze, and behavior in the health domain (Luo \& Isaacowitz, 2005) and are continuing to investigate the behavioral effects of motivated gaze toward healthrelevant information. Looking at looking not only provides an opportunity to link motivation, personality, development, and social psychology in the context of real-time transactions between the individual and the environment, but also has implications for designing visual stimuli (such as advertisements and health-promotion messages) intended to change behavior by grabbing people's gaze.

\section{Recommended Reading}

Isaacowitz, D.M. (2005). (See References)

Isaacowitz, D.M., et al. (in press). (See References)

Kleinke, C.L. (1986). (See References)

\section{REFERENCES}

Aspinwall, L.G., \& Brunhart, S.M. (1996). Distinguishing optimism from denial: Optimistic beliefs predict attention to health threats. Personality and Social Psychology Bulletin, 22, 993-1003.

Carstensen, L.L., Isaacowitz, D.M., \& Charles, S.T. (1999). Taking time seriously: A theory of socioemotional selectivity. American Psychologist, 54, 165-181.

Heckhausen, J., \& Schulz, R. (1995). A life-span theory of control. Psychological Review, 102, 284-304.

Isaacowitz, D.M. (2005). The gaze of the optimist. Personality and Social Psychology Bulletin, 31, 407-415. 
Isaacowitz, D.M., Wadlinger, H.A., Goren, D., \& Wilson, H.A. (in press). Selective preference in visual fixation to negative images in old age? An eye tracking study. Psychology and Aging.

Kleinke, C.L. (1986). Gaze and eye contact: A research review. Psychological Bulletin, 100, 78-100.

Kunda, Z. (1990). The case for motivated reasoning. Psychological Bulletin, 108, 480-498.

Light, J., \& Isaacowitz, D.M. (in press). The effect of developmental regulation on visual attention: The example of the "Biological Clock." Cognition and Emotion.

Luo, J., \& Isaacowitz, D.M. (2006). How optimists face skin cancer: Risk assessment, attention, memory, and behavior. Manuscript submitted for publication.

Mather, M., \& Knight, M. (2006). Angry faces get noticed quickly: Threat detection is not impaired among older adults. Journal of Gerontology: Psychological Sciences, 61, P54-P57.
Parkhurst, D., Law, K., \& Niebur, E. (2002). Modeling the role of salience in the allocation of overt visual attention. Vision Research, 42, $107-123$.

Pruzan, K., \& Isaacowitz, D.M. (2006). An attentional application of socioemotional selectivity theory in college students. Social Development, 26, 326-338.

Reichle, E.D., Pollatsek, A., Fisher, D.L., \& Rayner, K. (1998). Toward a model of eye movement control in reading. Psychological Review, 105, 125-157.

Wegener, D.T., \& Petty, R.E. (1994). Mood management across affective states: The hedonic contingency hypothesis. Journal of Personality and Social Psychology, 66, 1034-1048.

Xing, C., \& Isaacowitz, D.M. (2006). Aiming at happiness: How motivation affects attention to and memory for emotional images. Manuscript submitted for publication. 